Bundesgesundheitsbl -

Leitthema:Reisemedizin

I. Schöneberg · G. Rasch $\cdot$ L. Apitzsch

Robert Koch-Institut, Berlin

\title{
Reisebedingte Erkrankungen in Deutschland
}

\section{Ergebnisse der Einzelfall-Erhebungen des Robert Koch-Institutes}

\section{Zusammenfassung}

Personen, die internationale Grenzen überschreiten, sind in besonderem Maße gefährdet, Infektionskrankheiten zu erwerben. Eine Analyse des Auftretens gemeldeter übertragbarer Krankheiten in Deutschland zeigt: Eine große Zahl der Erkrankungen an Typhus, Paratyphus, Shigellose, Trichinose und Brucellose, die in den letzten Jahren in Deutschland zur Meldung kamen, wurde im Ausland erworben. Interventionsmaßnahmen sind vor allem bei den Infektionen des Magen-Darm-Traktes sowie bei den impfpräventablen Krankheiten notwendig.

\section{Schlüsselwörter}

Importierte Erkrankungen - meldepflichtige Krankheiten · Typhus · Paratyphus - Shigellose mportierte Erkrankungen haben in Deutschland hinsichtlich ihrer Aufmerksamkeit bei Bürgern, Medien und Öffentlichkeit einen hohen Stellenwert. Krankheiten können zum einen durch einreisende Ausländer nach Deutschland eingeschleppt werden, zum anderen sind es deutsche Bürger, die die Krankheiten bei einem Auslandsaufenthalt erwerben und nach Deutschland mitbringen.

\section{„Der größte Teil der nach Deutsch- land importierten Krankheiten wird gegenwärtig durch die Reisetätigkeit von Deutschen eingeschleppt."}

Früher spielten vor allem die Gastarbeiter eine wichtige Rolle beim Import bestimmter Krankheiten nach Deutschland. Heute sind es Asylbewerber, Flüchtlinge oder auch Besucher, die Infektionskrankheiten nach Deutschland einführen. Der größte Teil der importierten Krankheiten wird gegenwärtig jedoch durch Deutsche nach Deutschland eingeschleppt. Das betrifft in erster Linie deutsche Touristen, aber auch Geschäfts- und Dienstreisende. Da die Zahl der Reisenden ständig im Wachsen begriffen ist, kommt diesem Personenkreis - hinsichtlich notwendiger ProphylaxeMaßnahmen - auch zukünftig eine große Bedeutung zu.
Unter den importierten Erkrankungen ist nach wie vor die Malaria von besonderer Wichtigkeit, die mit jährlich ca. 800 bis 1000 Fällen in den letzten Jahren immer mit an vorderer Stelle lagen. Eine zusammenfassende Betrachtung für die Malariasituation in den letzten Jahren in Deutschland ist in einer gesonderten $\mathrm{Pu}$ blikation kürzlich dargestellt worden [1].

Waren einige der heute durch Reisen bedingten Erkrankungen schon immer mit einer Einschleppung aus fremden Ländern verbunden, so kam es bei einer Reihe weiterer Erkrankungen im Laufe der letzten Jahrzehnte zu einem Wandel. Früher war die Morbidität bestimmter Krankheiten in Deutschland so hoch, daß Importfälle nur eine untergeordnete Rolle spielten. Dazu gehören beispielsweise Typhus und Paratyphus, die besonders in den Nachkriegsjahren in Deutschland sehr häufig waren, aber auch die Shigellose. Heute sind diese Krankheiten in Deutschland selbst relativ selten, so daß Importfälle - mit einem Anteil von $50 \%$ und mehr - eine große Bedeutung erlangt haben.

Es gibt eine Reihe weiterer Erkrankungen, die zu einem mehr oder weniger großen Teil im Ausland erworben

Dr. Irene Schöneberg

Postfach 6502 80, D-13302 Berlin 
Bundesgesundheitsbl -

Gesundheitsforsch - Gesundheitsschutz

1999 - 42: 381-388 ㅇ Springer-Verlag 1999

I.Schöneberg · G. Rasch · L. Apitzsch

Travellers' diseases in Germany

\section{Summary}

Persons who cross international boundaries are at increased risk of contracting infectious diseases. An analysis of the reported communicable diseases in Germany shows: A large number of cases of typhoid fever, paratyphoid fever, shigella infections, trichinellosis and brucellosis notified during the last years in Germany originated from foreign countries. Intervention measures are necessary, above all, with regard to gastrointestinal infections and vaccine-preventable diseases.

\section{Key words}

Imported diseases - Notifiable diseases . Typhoid fever · Paratyphoid fever · Shigellosis

\section{Leitthema: Reisemedizin}

werden, bei denen wir den genauen Anteil der Importfälle jedoch nicht kennen. Hierzu gehören verschiedene Infektionen des Magen-Darm-Traktes, aber auch bestimmte Hepatitiden - insbesondere die Hepatitis A. Bei weiteren wichtigen Importkrankheiten - wie beispielsweise den Erkrankungen durch Dengue-Virus - wird gegenwärtig nicht einmal die Häufigkeit registriert.

\section{Erfassung reisebedingter Erkrankungen am Robert Koch-Institut (RKI)}

Die Erfassung einer Reihe von Krankheiten, darunter auch reisebedingte Erkrankungen, erfolgt laut Meldepflicht gemäß \$3 Bundes-Seuchengesetz (BSeuchG) im Rahmen von Meldungen an die Gesundheitsämter, die die aggregierten Daten über die Regierungsbezirke und Länder an das RKI weitergeben. Hier wird eine wöchentliche Statistik erstellt, die im „Epidemiologischen Bulletin“ regelmäßig veröffentlicht wird. Die im Rahmen der Meldepflicht erhobenen Daten sind auch die Grundlage für die Bundesstatistik, die vom Statistischen Bundesamt Wiesbaden erhoben wird [2]. Angaben zu Sterbefällen liegen für Deutschland überwiegend aus der Todesursachenstatistik vor [3]. Diese basiert auf der Auswertung der Totenscheine.

Für einige besonders wichtige Erkrankungen führt das RKI - mit Unterstützung der beteiligten Gesundheitsäm-

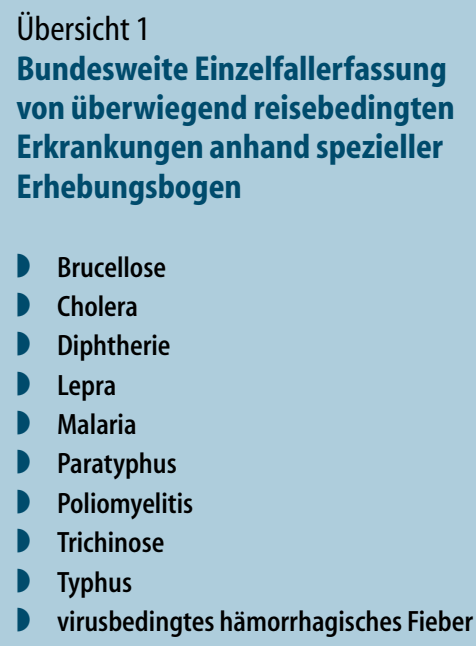

ter - Einzelfall-Erfassungen durch. Das betrifft für die Gruppe der reisebedingten Infektionskrankheiten die in der Übersicht 1 dargestellten Erkrankungen. Anhand spezieller Erhebungsbögen, die für alle in die Einzelfallerhebungen einbezogenen Erkrankungen erarbeitet wurden, werden detaillierte Angaben zum einzelnen Fall - in anonymisierter Form - an das RKI weitergegeben.

Neben den Angaben zur Malaria, die schon seit den 6oer Jahren erhoben werden, liegen im RKI damit für eine Reihe weiterer Krankheiten Ergebnisse aus Sondererhebungen vor. Die Erhebung der detaillierten Angaben zum Einzelfall erfolgt nicht routinemäßig und erfordert deshalb einen relativ hohen Aufwand. In den Gesundheitsämtern gestaltet sich die Zusammenstellung der Angaben teilweise sehr schwierig und langwierig, da zum einen die Mitarbeit verschiedener Ärzte, teilweise auch aus Kliniken und Labors, notwendig ist. Zum anderen erfordert das Ausfüllen der speziellen Erhebungsbögen eine umfangreiche Ermittlungstätigkeit zum einzelnen Erkrankungsfall.

Grundsätzlich muß davon ausgegangen werden, daß bei den im Rahmen von Sondererhebungen erfaßten Erkrankungen nur ein gewisser Ausschnitt abgebildet wird. Eine lückenlose Erfassung aller auftretenden Erkrankungen gelingt - wenn überhaupt - nur bei den wenigsten Infektionskrankheiten. Für einen kleinen Teil von Erkrankungen werden Sondererhebungen nur in einigen Bundesländern durchgeführt, d. h. für bestimmte Infektionskrankheiten werden Angaben zum Einzelfall in den neuen Bundesländern und Berlin gesondert erfaßt. Bei den Reisekrankheiten betrifft das die Shigellosen, aber auch beispielsweise die FSME. Für die FSME existiert allerdings zusätzlich ein spezielles Sentinel-System (s. u.).

\section{Häufigkeit des Vorkommens reisebedingter Erkrankungen in Deutschland}

Die reisebedingten Erkrankungen unterscheiden sich erwartungsgemäß hinsichtlich der Häufigkeit ihres Vorkommens in Deutschland. Kommt es bei ei- 
nigen der meldepflichtigen Infektionskrankheiten in Deutschland nur sehr selten zum Auftreten von Erkrankungen, so gibt es eine Reihe von Krankheiten, die in Größenordnungen von 1000 und mehr Erkrankungsfällen pro Jahr registriert werden. In Tabelle 1 sind für das Jahr 1997 die Anzahl der insgesamt gemeldeten Erkrankungsfälle sowie die Zahl der Erkrankungen, bezogen auf 100 ooo Einwohner, ausgewiesen.

Im folgenden sollen die im RKI vorliegenden Ergebnisse der bundesweiten Sondererhebungen (in der Reihenfolge der gemeldeten Häufigkeit ihres Auftretens) sowie die nur aus einzelnen Ländern übermittelten Angaben dargestellt werden.

\section{Typhus und Paratyphus}

Typhus und Paratyphus gehören zu den Krankheiten, die in früheren Jahren in größerem Ausmaß in Deutschland auftraten, heute jedoch nur noch in geringerer Anzahl erfaßt werden [2, 4]. Der Verlauf der Erkrankungszahlen beim Typhus, d. h. die früher und heute jährlich registrierten Fallzahlen, sind in Tabelle 2 dargestellt. Für die Erkrankungen an Typhus liegen Ergebnisse aus Einzelerfassungen anhand eines speziellen Bogens vor, die seit dem Jahr 1996 bundesweit erhoben werden. Die Auswertungen der detaillierten Daten zeigen, daß nur ein geringer Teil der in den Jahren 1996 und 1997 erfaßten Erkrankungen nicht im Ausland erworben wurde (Abb. 1).

Ein großer Teil der Erkrankungen trat nach Asienreisen auf, insbesondere nach Reisen durch Indien und Pakistan (31 bzw. 23 Erkrankungen). Auch Ägypten (20 Erkrankungen) und die Türkei (10 Erkrankungen) gehören zu den Reiseländern, aus denen Erkrankungen an Typhus in größerer Zahl eingeschleppt wurden.

Hinweise auf die Infektionsquelle liegen für den größten Teil der Fälle nicht vor. Auszugehen ist davon, daß insbesondere Wasser oder Lebensmittel Ausgangspunkt für die Infektionen waren. Aus den Sondererhebungen sind auch Angaben über durchgeführte Impfungen gegen Typhus bei den Erkrankten verfügbar. Danach erkrankten in den

Tabelle 1

In Deutschland gemeldete Erkrankungen 1997

Zahl der Erkrankungen
pro 100000 Einwohner

\begin{tabular}{llr}
\hline Shigellose & 2,4 & 1978 \\
Malaria & 1,2 & 1017 \\
Typhus & 0,1 & 75 \\
Paratyphus & 0,1 & 59 \\
Brucellose & 0,03 & 24 \\
Lepra & 0,007 & 6 \\
Cholera & 0,001 & 1 \\
Hepatitis A & 5,6 & 4614
\end{tabular}

Tabelle 2

Erkrankungen an Typhus in Deutschland - Zahl der gemeldeten Erkrankungen 1946-1996

\begin{tabular}{lrrr} 
Jahr & alte Bundesländer & neue Bundesländer & gesamt \\
\hline 1946 & 22406 & 36724 & 59130 \\
1956 & 2210 & 1707 & 3917 \\
1966 & 1015 & 359 & 1374 \\
1976 & 276 & 94 & 370 \\
1986 & 230 & 14 & 244 \\
1996 & 124 & 17 & 141
\end{tabular}

Jahren 1996/97 sechs Personen trotz vorheriger Impfung (das entspricht ca. 3\% der Erkrankten). Für Erkrankungen an Paratyphus ergibt sich hinsichtlich der Infektionsländer ein ähnliches Bild (Abb. 2). Länder Asiens und die Türkei liegen auch beim Paratyphus bei den Herkunftsländern der Infektionen an vorderer Stelle. So hatten im Jahr 199713 Erkrankungsfälle ihren Ursprung in Asien, 14 in der Türkei und vier in anderen europäischen Ländern.

\section{Brucellose}

Auch bei der Brucellose kam es in den zurückliegenden Jahren zu gravierenden Veränderungen[5]. Dies betrifft zum einen die Zahl der auftretenden Erkrankungen (Abb. 3), zum anderen die Herkunft der Infektionen. Früher bestand in Deutschland eine enge Verbindung zwi- schen den Infektionen beim Tier und den Erkrankungen des Menschen. Im Zusammenhang mit der Sanierung der deutschen Tierbestände kam es zu immer weniger Erkrankungen, die auf den Kontakt zu einheimischen Tieren zurückgeführt werden konnten. Heute werden Erkrankungen an Brucellose zu einem großen Teil durch Lebensmittel verursacht, die ihren Ursprung im Ausland - oft in Ländern des südlichen Europa - haben, oder dort verzehrt wurden (Abb. 4). Insgesamt 17 Erkrankungen wurden von 1995 bis 1997 aus der Türkei importiert. Weitere Importländer waren unter anderem Irak (vier), Kasachstan (zwei), Frankreich (zwei), Mazedonien, Syrien (zwei), Spanien (zwei) und Portugal (drei). Einzelne Erkrankungen hatten ihren Ursprung jedoch auch in Afrika (Kenia, Marokko, Somalia) oder in Amerika. 

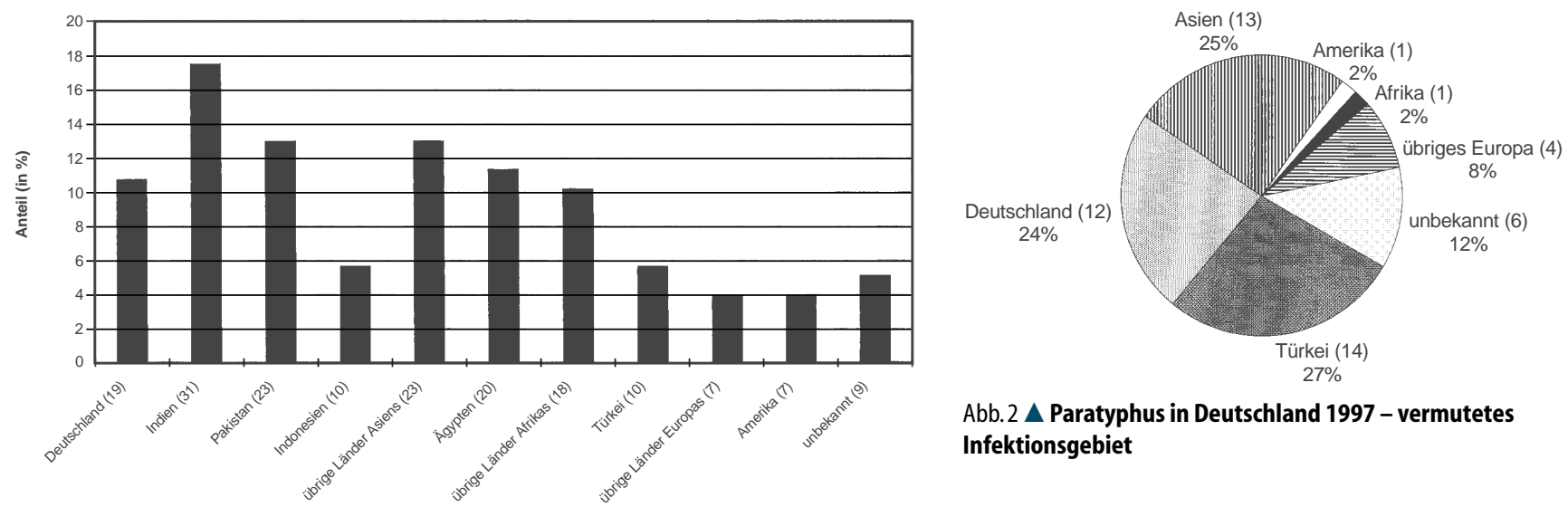

Abb. $2 \triangle$ Paratyphus in Deutschland 1997 - vermutetes Infektionsgebiet

Abb. 1 A Typhus in Deutschland 1996/97 - vermutetes Land der Infektion
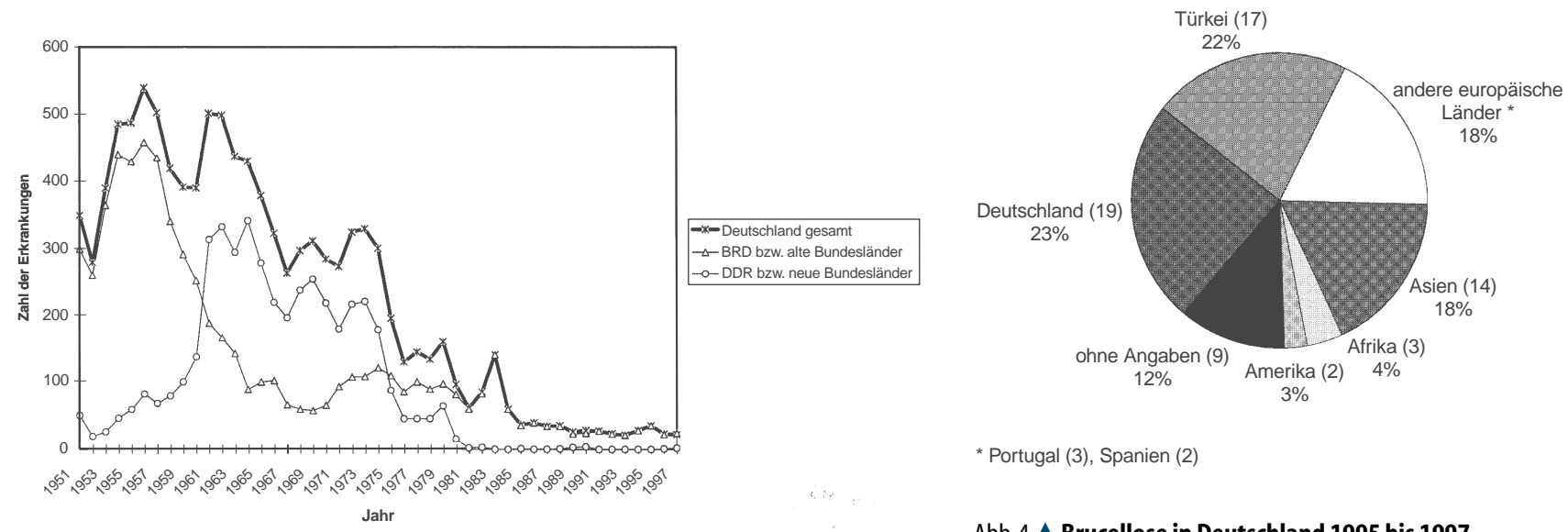

* Portugal (3), Spanien (2)

Abb. $3 \Delta$ Erkrankungen an Brucellose in Deutschland 1951 bis 1997

Abb. $4 \triangle$ Brucellose in Deutschland 1995 bis 1997 vermutetes Land der Infektion

Trichinose

Erkrankungen an Trichinose wurden in den Jahren von 1991 bis 1994 in Deutschland nur in geringer Fallzahl registriert (jährlich bis zu drei Erkrankungen). 1995 kam es zu zehn gemeldeten TrichinoseErkrankungen, 1996 zu einer, 1997 zu neun Erkrankungen. Die Ergebnisse der Sondererhebungen (seit 1995) zeigen, daß Erkrankungen an Trichinose in Deutschland heute ganz überwiegend aus anderen Ländern importiert werden. Der größte Teil der Infektionen wurde von Bürgern, die ursprünglich aus Ländern des Balkan stammten, anläßlich von Heimatbesuchen erworben (Tabelle 3 ).

\section{Lepra}

In den letzten Jahren kam es immer wieder zu einzelnen Erkrankungen an Lepra. Im Zeitraum der Jahre von 1990 bis 1997 waren das jährlich zwischen einer (1992) und sieben (1990) Erkrankungen. Betroffen von den Lepra-Erkrankungen sind fast ausschließlich Bürger anderer Länder, die zum größten Teil aus Endemiegebieten stammten. Die Erkrankungen an Lepra, die 1997 in Deutschland zur Meldung kamen, sind in Tabelle 4 dargestellt.

\section{Poliomyelitis}

Die Poliomyelitis gehörte bis 1961 zu den Erkrankungen, die in Deutschland in Größenordnungen von einigen tausend
Erkrankungsfällen auftraten. Mit der Einführung der Schutzimpfung und dem damit verbundenen Rückgang der Erkrankungszahlen kam es dazu, daß Erkrankungen an Poliomyelitis zu einem größeren Teil aus anderen Ländern importiert wurden [6]. In den 7oer Jahren machten nach Deutschland mitgebrachte Erkrankungen den größten Teil der in Deutschland gemeldeten Fälle an Poliomyelitis aus [7].

In den letzten Jahren waren die in Deutschland erfaßten Erkrankungen an Poliomyelitis fast ausschließlich Vakzine-assoziiert. Die letzten zwei bekannten Fälle von Poliomyelitis durch importierte Wildviren traten im Jahre 1992 auf (aus Ägypten bzw. Indien). Davor kam es $1990 \mathrm{zu}$ Poliomyelitiserkrankungen bei einem Kleinkind aus Angola (nach 
Reise nach Angola) und bei einem türkischen Kind (nach Reise in die Türkei). 1994 wurde eine Vakzine-assoziierte Poliomyelitiserkrankung gemeldet, die in der Türkei ihren Ursprung hatte. Das Virus konnte bei seiner Differenzierung eindeutig als Impfvirus bestimmt werden.

\section{Cholera}

Erkrankungen an Cholera kamen in den zurückliegenden Jahren in Deutschland nur in wenigen Fällen zur Meldung. Nachdem im Jahre 1995 ein 67jähriger Mann nach Aufenthalt in Bali erkrankt war, wurde 1996 in Deutschland keine Cholera-Erkrankung gemeldet. 1997 erkrankte eine 41jährige Frau nach Aufenthalt in Tunesien an Cholera. Für beide Erkrankungsfälle konnte der Stamm im Referenzlabor als Vibrio cholerae O1, Serotyp Ogawa, Biotyp El Tor bestätigt werden. Im Frühjahr des Jahres 1998 kam es - im Zusammenhang mit Überschwemmungen in Kenia - innerhalb eines kurzen Zeitraumes (Januar, Februar) zu insgesamt fünf Importfällen bei deutschen Touristen (ebenfalls Vibrio cholerae O1, Serotyp Ogawa, Biotyp El Tor). Der neue Typ O 139 trat bisher nur in einem Fall 1993 in Deutschland auf [8]. Betroffen war ein in Deutschland lebender Pakistani, der nach Rückkehr von einem Heimaturlaub erkrankte.

\section{Tollwut}

Auch andere seltene Erkrankungen wie die Tollwut werden sehr gründlich recherchiert, wenn auch kein spezieller Erhebungsbogen hierfür vorliegt. Der letzte gemeldete Fall einer Erkrankung an Tollwut war 1996. Er trat nach einem Hundebiß in Sri Lanka auf.

\section{Shigellose}

Von der Zahl der in Deutschland jährlich registrierten Erkrankungen steht die Shigellose bei den reisebedingten Infektionskrankheiten heute mit an vorderer Stelle. Traten in früheren Jahren Erkrankungen an Shigellose in Größenordnungen von zigtausenden von Fällen auf, so wurden im Jahre 1997 in Deutsch- land insgesamt nur noch 1978 Erkrankungen gemeldet (Abb. 5). Für die Shigellosen sind Angaben zu den einzelnen Erkrankungsfällen nur aus den neuen Ländern und Berlin verfügbar. Hier kam es im Jahre $1997 \mathrm{zu} 751$ erfaßten Erkrankungen. $75 \%$ dieser Fälle wurden im Ausland erworben. $\mathrm{Zu}$ den wichtigsten Importländern für die Shigellose im Jahre 1997 zählten Ägypten, Tunesien, die Türkei, Marokko und die Dominikanische Republik (Tabelle 5). Differenzierte Angaben zum Erreger zeigen, daß der größte Teil der Infektionen durch Sh. sonnei $(81 \%)$ verursacht wurde (Tabelle 6). Während Infektionen durch Sh. flexneri in immerhin $17 \%$ der Fälle auftraten, wurden Sh. boydii bzw. Sh. dysenteriae nur in einzelnen Fällen (sieben bzw. sechs Fälle) registriert.

\section{Zusammenfassende Bewertung}

Reisebedingte Erkrankungen sind für den Epidemiologen von besonderem Interesse, weil sich aus der Sammlung und Analyse entsprechender Daten zum einen die bestmöglichen Präventionsmaßnahmen vor beabsichtigten Reisen

\begin{tabular}{llll}
\multicolumn{2}{l}{$\begin{array}{l}\text { Tabelle } 3 \\
\text { Registrierte Erkrankungen an Trichinose in Deutschland }\end{array}$} & \\
Jahr & Anzahl der Erkrankungen & Infektionsgebiet & Tierart \\
\hline 1996 & 1 & Deutschland & Schwein \\
1997 & 9 & $\begin{array}{l}\text { Rumänien:6 Fälle } \\
\text { Restjugoslawien:1 Fall } \\
\text { Deutschland:2 Fälle }\end{array}$ & Schwein/Wildschwein \\
& & &
\end{tabular}

Tabelle 4

In Deutschland 1997 diagnostizierte Erkrankungen an Lepra

\begin{tabular}{|c|c|c|}
\hline $\begin{array}{l}\text { Herkunft des } \\
\text { Erkrankten }\end{array}$ & $\begin{array}{l}\text { Wahrscheinliches } \\
\text { Infektionsgebiet }\end{array}$ & Typ \\
\hline Engländerin, 53 J.w. & Indien & Tuberkuloider Typ \\
\hline Äthiopierin, 16 J.w. & Äthiopien & Lepromatöser Typ \\
\hline Bürger Vietnams, 32 J.m. & Vietnam & Lepromatöser Typ \\
\hline Bürger Hongkongs, $57 \mathrm{~J} . \mathrm{m}$. & Taiwan & Lepromatöser Typ/Borderline Typ \\
\hline Bürger Pakistans, 31 J.m. & Pakistan & Tuberkuloider Typ \\
\hline Bürger Indiens, 39 J.m. & Indien & Borderline Typ \\
\hline
\end{tabular}

und Aufenthalten in entsprechenden Regionen (Maßnahmen der Expositionsprophylaxe, Schutzimpfungen oder eine Chemoprophylaxe) ableiten lassen. Zum anderen ist das Interesse aber auch begründet durch Befürchtungen einer Weiterverbreitung dieser Erkrankungen in Deutschland nach ihrer Einschleppung und die Notwendigkeit, Vorsorgemaßnahmen hierfür vorzusehen.

Bei den von Importkrankheiten betroffenen Personen handelt es sich den Daten der Einzelfall-Erfassungen für verschiedene Krankheiten zufolge zu einem großen Teil um Deutsche, denen hinsichtlich der Prävention bei Reisen in andere geographische Regionen eine besondere Aufmerksamkeit gewidmet werden sollte. Wichtig für den Reisenden ist in diesem Zusammenhang eine optimale Impfprophylaxe, die die Gegebenheiten des besuchten Landes und die Art der Reise ebenso berücksichtigt (z. B. Typhus, Hepatitis A, Hepatitis B, Poliomyelitis, FSME, Gelbfieber, Cholera usw.) wie den auch in Deutschland generell empfohlenen Impfschutz (z. B. Tetanus, Diphtherie, Masern-MumpsRöteln usw.). 


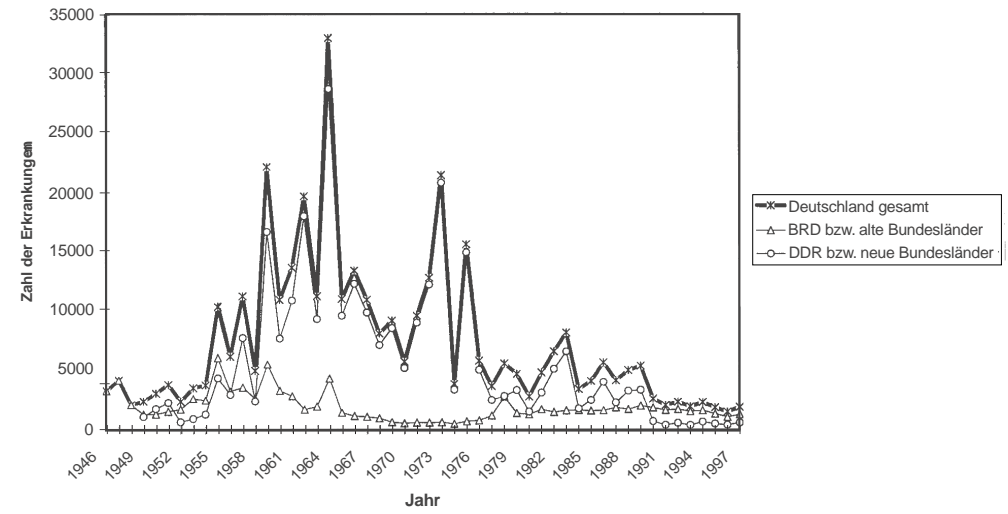

Abb. $5 \Delta$ Erkrankungen an Shigellose in Deutschland 1946 bis 1997

\section{„Die Prävention reisebedingter Erkrankungen basiert auf Maßnahmen der Expositions- prophylaxe, aufSchutzimpfungen und der Chemoprophylaxe."}

Daneben stellt die Chemoprophylaxe zur Malaria-Vorbeugung eine wichtige Komponente des Gesundheitsschutzes für Reisen in Endemiegebiete dar.

Eine große Bedeutung kommt der Frage der Expositionsprophylaxe zu. Insbesondere bei Infektionskrankheiten, die auf fäkal-oralem Wege übertragen werden können, sind Maßnahmen der Expositionsprophylaxe gut geeignet, das Risiko von Infektionen gering $\mathrm{zu}$ halten. Beispielsweise sollte der Verzehr roher Speisen, direkt vom Straßenhändler gekauft, in Ländern mit mangelhafvermieden werden wie die Verwendung unabgekochten Wassers. Bei Krankheiten, die durch Insekten übertragen werden, sollte Maßnahmen, die der Verhinße Aufmerksamkeit geschenkt werden (Repellents, Moskitonetz usw.). Informationsschriften sowie die reisemedizinische Beratung sollten die Problematik der Expositionsprophylaxe in geeigneter Weise berücksichtigen.

Leider gestatten die erhobenen Daten zu den reisebedingten Erkrankungen keine Aussage zur Art der durchgesacktourismus“, Geschäftsreise), so daß weitergehende Analysen in diesem $\mathrm{Zu}$ ten hygienischen Bedingungen ebenso derung von Insektenstichen dienen, groführten Reise (Pauschalurlaub, „Ruck- sammenhang nicht möglich sind. Lediglich zur Malaria sind besser differenzierte Angaben verfügbar [1].

Mit den Angaben aus der EinzelfallErfassung liegen auch Daten vor, die Rückschlüsse auf Kontakterkrankungen bzw. eine mögliche Weiterverbreitung in
Deutschland zulassen. In keinem Fall kam es beispielsweise bei den CholeraErkrankungen der letzten Jahre zu einem Sekundärfall. Ein besonderes Problem hinsichtlich der nötigen einzuhaltenden Sicherheitsmaßnahmen stellen auch die virusbedingten hämorrhagischen Fieber dar. Außer Einzelfällen von Dengue Hämorrhagischem Fieber, das jedoch im Normalfall nicht direkt von Mensch zu Mensch übertragen wird, wurden in den letzten Jahren keine Importe anderer Erkrankungen aus dieser Gruppe (wie Ebola, Lassa usw.) nach Deutschland bekannt (Dengue Hämorrhagisches Fieber: 1996 kein Fall, 1997 ein Fall nach Aufenthalt in Thailand gemeldet).

Bei den Shigellosen wurden im Rahmen der Einzelfallerfassung in den neuen Bundesländern und Berlin auch Kontakterkrankungen zu Importfällen in Deutschland erfaßt. Es handelte sich 1997 insgesamt um sieben Häufungen
Tabelle 5

Gemeldete Erkrankungen an Shigellose in Deutschland 1997 -

Herkunft der Infektionen (neue Bundesländer und Berlin)

Zahl der Erkrankungen: 1978, davon in den neuen Bundesländern und Berlin: 751, davon Importfälle: 566

Vermutetes Land der Infektion:

\begin{tabular}{lcll}
\hline Ägypten & 157 Fälle & Dominikanische Republik & 29 Fälle \\
Tunesien & 120 Fälle & Kenia & 16 Fälle \\
Türkei & 78 Fälle & Indien & 12 Fälle \\
Marokko & 32 Fälle & Bulgarien & 12 Fälle
\end{tabular}

Tabelle 6

Gemeldete Erkrankungen an Shigellose in Deutschland 1997 -

Erregerspezies (neue Bundesländer und Berlin)

Zahl der Erkrankungen: 1978, davon in den neuen Bundesländern und Berlin: 751, davon Importfälle: 566

Verteilung der Spezies:
Sh. sonnei

Sh. flexneri

Sh. dysenteriae

Sh. boydii
607 Fälle $(81 \%)$

131 Fälle (17\%)

7 Fälle (1\%)

6 Fälle (1\%)
$438(77 \%)$

$117(21 \%)$

$7(1 \%)$

$4(1 \%)$ 
(mit insgesamt 55 Erkrankungen), die in Reisegruppen bzw. Familien während und nach Aufenthalten in Ägypten, der Türkei, in Tunesien, Bulgarien und Griechenland auftraten. Von diesen 55 Erkrankungen konnten 29 Fälle als Kontaktfälle in Deutschland zugeordnet werden. Diese Zahl ist im Vergleich zu den importierten Erkrankungen (insgesamt 566) und zu den in früheren Jahren beobachteten Fallzahlen eher gering.

Bei den Hepatitiden insgesamt ist mit einem erheblichen Anteil von importierten Erkrankungsfällen zu rechnen. Das betrifft vor allem die Hepatitis A, wo Schätzungen von $50 \%$ und mehr Importfällen ausgehen. Die Hepatitis A ist heute die häufigste durch Impfung verhütbare Infektionskrankheit bei Reisenden.

Für die Hepatitis A gibt es bisher keine Daten zum Einzelfall, also auch keine Angaben zur Infektionsquelle und zur Herkunft. Lediglich im Ausnahmefall - bei Auftreten von größeren Häufungen - gelangen Berichte darüber zum RKI. Auch bei der Hepatitis A treten Kontakterkrankungen auf, über die jedoch wenig konkrete Informationen vorliegen. Lebensmittel-bedingte Häufungen, bei denen für den Indexfall eine Reise bekannt war, wurden in der Vergangenheit beschrieben [9-11].

Die Bedeutung von Reisen für das Auftreten von Hepatitis A ist auch aus dem jahreszeitlichen Verlauf der Erkrankungsfälle ablesbar. Im dritten Quartal, nach dem Ende der Mehrzahl der Sommerreisen, ergab sich in allen Jahren ein Anstieg der gemeldeten Fälle. Dieser fiel in den letzten Jahren jedoch geringer als in früheren Jahren aus, was möglicherweise als ein Hinweis auf eine bessere Inanspruchnahme von Schutzimpfungen vor Reisen zu werten ist.

Auch bei der Hepatitis B ist davon auszugehen, daß ein Teil der Erkrankungen im Ausland erworben wird. Wie hoch dieser Anteil ist, kann jedoch nur geschätzt bzw. vermutet werden. Für Deutschland liegen hierzu lediglich Daten aus der ehemaligen DDR (von 1989) vor, die die heutige Situation sicherlich nicht widerspiegeln können [12].

Für andere Erkrankungen, die nicht im Katalog der meldepflichtigen Krank- heiten enthalten sind, liegen Daten und Angaben zum Einzelfall nicht vor. Dies betrifft z.B. auch Infektionen durch das Dengue-Virus. Schätzungen für Deutschland gehen von mehr als 1000 Erkrankungen pro Jahr aus, aber nur hämorrhagische Verläufe sind meldepflichtig.

Das trifft auch für die Hepatitis E $\mathrm{zu}$, die in die Kategorie Hepatitis, übrige Formen, eingeht, nicht aber speziell ausgewiesen wird und in gewissem Maße auch für die FSME, die zwar zahlenmäßig in der Meldekategorie Virus-Meningoenzephalitis erfaßt wird, nicht jedoch in einer gesonderten Kategorie. Aus den neuen Bundesländern und Berlin liegen Daten zu den Einzelfällen an FSME vor. Diese gestatten jedoch nicht in jedem Fall exakte Rückschlüsse auf den Ort der Infektion. Nicht für jede der erfaßten Erkrankungen war ein Zeckenstich erinnerlich. Ebenso waren Angaben zu Reisen nicht in jedem Fall ermittelt worden. So konnten für einen Teil der Erkrankungen Gebiete Deutschlands als Herkunftsort zugeordnet werden (vor allem in Bayern oder Baden-Württemberg). Für einen anderen Teil der Fälle wurden Österreich, Tschechien, die Slowakei oder weitere Länder angegeben.

\section{"Für eine Reihe von Erkrankungen, die fast ausschließlich (Dengue) oder überwiegend nach Deutschland importiert werden (Hepatitis $A / E$, Shigellose), liegen keine oder nur unzureichende Meldedaten vor."}

Anhaltspunkte zum Anteil importierter Erkrankungen bei der FSME in Deutschland erbrachten Daten, die im Rahmen eines Sentinels im süddeutschen Raum - in Zusammenarbeit mit Kliniken und Gesundheitsämtern - erhoben (Prof. Roggendorf, Frau Dr. Jäger, PD Dr. Kaiser) und dem Robert KochInstitut zur Verfügung gestellt wurden. Von 1974 bis 1997 wurden insgesamt 1462 Erkrankungen an FSME in diesem $\mathrm{Zu}$ sammenhang erfaßt. Die Auswertung ergab, daß nur bei 84 Personen eine Infektion im Ausland angenommen wird, was ca. $6 \%$ entspricht. Bei den Herkunftsländern steht Österreich (34) an der Spitze. Andere Länder waren nur jeweils ein- oder zweimal vertreten.

$\mathrm{Zu}$ den Erkrankungen, die heute vermehrt durch Bürger anderer Länder nach Deutschland gebracht werden, gehört die Tuberkulose [13]. Das betrifft, neben den schon länger in Deutschland lebenden Ausländern, vor allem Asylbewerber und Kriegsflüchtlinge. Betroffen sind aber auch Aussiedler, die aus osteuropäischen Ländern mit hoher Tuberkulose-Inzidenz nach Deutschland kommen, beispielsweise aus den Nachfolgestaaten der ehemaligen Sowjetunion.

Aus den dargestellten Ergebnissen der Sondererhebungen wird deutlich, daß für eine Reihe wichtiger, im Rahmen der Meldepflicht genannter Erkrankungen, detaillierte Angaben zum Einzelfall im RKI vorliegen. Das trifft auf alle Krankheiten zu, die in die Sondererhebungen einbezogen wurden (Übersicht 1).

Einschränkend muß man feststellen, daß nur die Daten in die Auswertungen und Betrachtungen im RKI einbezogen werden können, die in die Meldungen nach Bundes-Seuchengesetz eingegangen sind. Des Weiteren ist unbekannt, in welchem Maße die erhobenen Daten bei den einzelnen Krankheiten den tatsächlich vorkommenden Erkrankungszahlen entsprechen. Erwähnt werden muß in diesem Zusammenhang das Problem der Untererfassung, das mit Sicherheit auch für die reisebedingten Erkrankungen relevant ist. Das Ausmaß der Untererfassung ist für die einzelnen Erkrankungen sicherlich unterschiedlich, jedoch bisher nur schwer abzuschätzen.

Zwangsläufig ergeben sich deshalb bei der Interpretation der erhobenen Daten gewisse Einschränkungen. Es versteht sich deshalb von selbst, daß eine Ausdehnung der Meldepflicht auf weitere Erkrankungen, wie sie bei der Neufassung des Infektionsschutzgesetzes möglich wäre, allein nicht zu einer spürbaren Verbesserung der Surveillance führen würde. Notwendig erscheint es vielmehr, in Zukunft weitere Erfassungsmethoden zu etablieren, die dazu beitragen, die durch die Meldepflicht gewonnenen Ergebnisse zu überprüfen bzw. zu ergänzen. 


\section{Literatur}

1. Schöneberg I,Apitzsch L, Rasch G (1998) Malaria - Erkrankungen und Sterbefälle in Deutschland 1993 bis 1997. Das Gesundheitswesen 60:755-761

2. Statistisches Bundesamt Gesundheitswesen. Fachserie 12, Reihe 2: Meldepflichtige Krankheiten (Wiesbaden 1990-1996)

3. Statistisches Bundesamt Gesundheitswesen. Fachserie 12, Reihe 4: Todesursachen (Wiesbaden 1990-1996)

4. Pöhn HP, Rasch G (1994) Statistik meldepflichtiger übertragbarer Krankheiten. BGA-Schriften 5/93. München:MMV Medizin Verlag

5. Rasch G, Schöneberg I, Apitzsch L, Menzel U (1997) Brucellose-Erkrankungen in Deutschland. Bundesgesundhbl 40:50-54

6. Weise HJ, Pöhn HPh (1984) Epidemiologie der Poliomyelitis in der Bundesrepublik Deutschland und Berlin (West) 1978 bis 1982. Münch med Wschr 126:269-274

7. Weise HJ (1976) Tourismus und Infektionsrisiko. Münchn med Wschr 118: 1061-1068

8. Rasch G (1996) Importierte Infektionskrankheiten. Bundesgesundhbl 39:228-234

9. Becker B, Prömse B, Krämer J, Exner M (1996) Übertragung humanpathogener Viren durch Lebensmittel: Hepatitis-A-Epidemie ausgelöst durch Backwaren im Kreis Euskirchen (NRW). Das Gesundheitswesen 6:339-340

10. Robert Koch-Institut Hepatitis-A-Häufungen durch kontaminierte Lebensmittel. Epidemiologisches Bulletin 39/96

11. Robert Koch-Institut Hepatitis-A-Ausbruch in Nordbayern. Epidemiologisches Bulletin 30/98

12. Hallauer JF, Rasch G (1992) Epidemiology of hepatitis B in Germany. In: Bennett DL (ed) The control of hepatitis B:The role of prevention in adolescence. London: Gower Medical Publishing, 47-52

13. Deutsches Zentralkomitee zur Bekämpfung der Tuberkulose 23. Informationsbericht. Berlin 1997. Frankfurt am Main:pmi Verlagsgruppe GmbH Frankfurt am Main
In den vergangenen Wochen erreichten uns die unten aufgeführten Neuankündigungen. Ausgewählte Titel werden in nächster Zeit besprochen.

\section{K. Bork \\ Arzneimittelnebenwirkungen \\ an der Haut}

2., überar. u. erw. Aufl.; Stuttgart, New York: Schattauer, 1999. 416 S., 408 mehrfarb. Abb., 90 Tab., (ISBN 3-7945-1860-8), geb., DM 198,-

U. Quast

100 und mehr knifflige Impffragen 4., überar. u. erw. Aufl.; Stuttgart: Hippokrates, 1998.252 S., 5 Abb., 9 Tab., (ISBN 3-7773-1254-1), kart., DM 39,-

\section{H.-J.Tietz, H. Ulbricht}

Humanpathogene Pilze der Haut und Schleimhäute

Hannover: Schlütersche, 1999. 192 S.,

139 farb. Abb., 94 Zeichn., 4 Tab.,

(ISBN 3-87706-540-6), DM 198,-

\section{H. A. Dieterich, H. G. Eichler, A. Kurz}

Antiinfektiva

Stuttgart:WVG, 1998.220 S., 66 Abb., 30 Tab., (ISBN 3-8047-1498-6), kart., DM 56,-

\section{N. Peseschkian}

Psychosomatik und Positive

Psychotherapie

Stuttgart, Jena, Lübeck: Fischer, 1999. 589 S.,

(ISBN 3-596-11713-5), kart., DM 22,90

\section{H.J.Moriske, E. Turowski}

Handbuch für Bioklima und Lufthygiene Landsberg:ecomed, 1998. Ca. 400 S. Loseblatt, (ISBN 3-60972580-X), Leinenordner,DM 178,-

H. Remschmidt

Praxis der Psychotherapie mit Kindern und Jugendlichen

Köln:Deutscher Ärzte-VIg., 1998. 172 S., 21 Abb. 28 Tab., (ISBN 3-7691-0371-8), brosch., DM 68,K. U.Tuch, C. Borchard-Tuch

\section{Anästhesie (essentials)}

Intensivkurs der Weiterbildung

2., überar. u.erw. Aufl.; Stuttgart: Enke, 1999. 394 S., 22 Abb., 117 Tab.,(ISBN 3-432-27012-7), kart., DM 98,-

A. Hüter-Becker

Biomechanik, Arbeitsmedizin, Ergonomie

Stuttgart, New York:Thieme, 1998.524 S., 292

Abb., 47 Tab., (ISBN 3-13-101251-X/692),

kart., DM 54,-

W. Jilg, S. Dittmann
Lern- und

Informationssystem

CD-ROM

Impfen

Stuttgart, New York: Thieme, 1998. 2 CD-ROMs mit Booklet, (ISBN 3-13-104841-7/696),

DM 148,-

\section{A. Hofstetter}

Urogenitale Infektionen

Berlin, Heidelberg, New York: Springer, 1999. 602 S., 163 Abb., 99 Tab., (ISBN 3-540-64173-4) geb., DM 198,-

\section{G. Stux, N. Stiller, B. Pomeranz}

Akupunktur

5., überar. u. erw. Aufl.; Berlin, Heidelberg, New York:Springer, 1999.427S., 134 Abb., 3 Farbtaf., (ISBN 3-540-64997-2), geb., DM 179,-

\section{G. Stux}

Einführung in die Akupunktur

Berlin, Heidelberg, New York: Springer, 1999.

322 S., 64 Abb., 19 Tab., (ISBN 3-540-64568-3)

brosch., DM 39,90

\section{U.Placzek}

Menschen, Medien, Medizin

Ein Frauenarzt nimmt Stellung

Gelnhausen:TRIGA, 1998.290 S.,

(ISBN 3-931559-80-7), Pb., DM 29,80

\section{U. Knapp}

Die Wunde

2., komplett überarb. u. erw. Aufl.; Stuttgart, New York:Thieme, 1999.229 S., 178 Abb. in 302 Einzeldarst., 11 Tab., (ISBN 3-13-603202-0/694), geb., DM 168,-

\section{H.-J.Schade}

Das Handbuch der Selbstzahlerpraxis Landsberg: ecomed, 1999.273 S., (ISBN 3-609-51340-3), geb., DM 78,-

\section{F. Hofmann}

Impfen

5., überarb. u. erw. Aufl.; Landsberg:ecomed, 1999. 224 S., (ISBN 3-609-63185-6), Pb., DM 24,80

\section{R. Gross, P. Schölmerich, W. Gerok}

Die Innere Medizin

Classics-Ausgabe der 9., neu bearb. Aufl.; Stuttgart, New York: Schattauer, 1999. 1400 S., 630 meist farb. Abb., 72 farb. Abb.auf 12 Taf., 565 Tab., 62 Synopsen, (ISBN 3-7945-1900-0), geb., DM 118,-

\section{G. Paumgartner}

Therapie Innerer Krankheiten

Berlin, Heidelberg, New York: Springer, 1999. 1720 S., 148 Abb., 647 Tab., (ISBN 3-540-64580-2), geb., DM 298,- 